\title{
Spontaneous CSF Leaks and Meningoencephaloceles: Endoscopic Repair and Possible Etiology
}

\author{
${ }^{1}$ Andrey S Lopatin, ${ }^{2}$ Dmitry N Kapitanov, ${ }^{2}$ Alexander A Potapov \\ ${ }^{1}$ Ear, Nose and Throat Clinic, First Sechenov Moscow Medical University, Moscow, Russia \\ ${ }^{2}$ Burdenko Institute of Neurosurgery, Russian Academy of Medical Science, Moscow, Russia
}

Correspondence: Andrey S Lopatin, Ear, Nose and Throat Clinic, First Sechenov Bolshaya Pirogovskaya 6, Bld 1, 119992 Moscow, Russia, e-mail: lopatin@mma.ru

\section{ABSTRACT}

Objective: To assess the outcomes of endonasal endoscopic repair of spontaneous cerebrospinal fluid (CSF) rhinorrhea and to analyze its possible etiological factors.

Patients: During the period between J anuary 1999 and November 2011, 173 patients who presented with spontaneous CSF rhinorrhea underwent endonasal endoscopic surgery. Preoperative examination included CT scans, nasal endoscopy, measurement of glucose concentration in the nasal discharge and, in some cases, cisternographic evaluation via CT or MRI. CSF fistula closure was performed using endonasal endoscopic technique under general anesthesia. In all, 186 surgeries (173 primary attempts and 13 revisions) were carried out over the 12-year period. A combination of different plastic materials, i.e. nasal septum cartilage, facia lata, abdominal fat, rotating middle turbinate flaps, and fibrin glue was used for fistula repair.

Results: At the time of the surgery, sites of the CSF fistula were determined as follows: Cribriform plate- 70 , fovea ethmoidalis-55, sphenoid sinus -45 , frontal sinus - 3. Extremely pneumatized lateral extension of the sphenoid sinus was found in 26 patients, and a meningo/encephalocele protruding through the bony defect was the source of the leak in 23 of them. In terms of up to 11 years, 165 patients were treated successfully after first attempt and five more recovered after revision endoscopic surgery. Success rate after the first surgery was $95.4 \%$, overall success rate- $97.7 \%$. There were no postoperative complications.

Conclusion: Possible etiological factors of this disease include obesity, innate skull base malformations, overpneumatized sphenoid sinus, particularly presence of its lateral extensions and the empty sella syndrome. Endoscopic endonasal repair of spontaneous CSF rhinorrhea appears to be a safe and successful procedure. However, technique of endoscopic closure of CSF fistulas in the lateral part of the sphenoid sinus needs further perfection.

Keywords: Spontaneous cerebrospinal fluid leaks, Endoscopic skull base surgery, Sphenoid sinus.

\section{INTRODUCTION}

Persistent cerebrospinal fluid (CSF) leaks are usually considered primary (spontaneous) or secondary. Secondary CSF leaks are rather common and they accompany $80 \%$ of severe head trauma. A specific form of the secondary traumatic CSF leak is iatrogenic liquorrhea, which occurs not infrequently during skull base and endonasal sinus surgery. Secondary CSF rhinorrhea can be caused by skull base and intracranial tumors: A ngiofibromas, osteomas, etc. destroying skull base bones. In contrast, primary (spontaneous) CSF rhinorrhea has been usually recognized as a rare condition presenting both diagnostic and therapeutic challenges.

Development of endonasal endoscopic surgery has made endonasal procedures less invasive and significantly improved results of CSF rhinorrhea therapy. CSF rhinorrhea is now one of its well-established extended indications. ${ }^{1,2}$ In most cases, endoscopic endonasal technique for CSF leak repair has almost completely replaced more traumatic transcranial and extracranial procedures which had been widely used before. However, methods for the correct location and techniques for surgical closure of spontaneous CSF fistulas still need further perfectioning.

\section{PATIENTS AND METHODS}

During the period from J anuary 1999 to N ovember 2011, 173 patients with confirmed diagnosis of spontaneous CSF rhinorrhea were admitted, all of them underwent endonasal endoscopic surgery and they formed our study group. There were 143 women and 30 men aged between 11 months and 72 years old. Of the 173 patients, 164 had both anterior and postnasal CSF discharge, whereas nine patients who did not have release of CSF from the nostrils experienced permanent cumbersome leakage from the nasal cavity onto the posterior pharyngeal wall which caused irritation and cough.

Thirty one patients from this series experienced at least one episode of meningitis, 11 patients reported recurrent episodes. Pneumatocephalus was revealed in two cases. Duration of the condition, i.e. the period between first presentation of the CSF leak and admittance in the clinic was less than 2 months in 22 cases, between 2 and 6 months 
- in 57 cases, between 6 and 12 months- in 21 cases. Seventy-three patients experienced liquorrhea for more than year. Surgical fistula closure had previously been attempted in 15 cases. Of these, six patients operated on with lumboperitoneal shunting and two had surgeries via transcranial approach. The other seven patients underwent attempts of endonasal fistula closure. N one of the attempts achieved steady positive result.

Preoperative examination included high-resolution CT scans of the paranasal sinuses and skull base, nasal endoscopy and evaluation of glucose concentration in the nasal discharge. When initial CT scans did not identify a bone defect in the skull base, patients underwent cisternographic evaluation via CT and/or M RI. Preoperative nasal endoscopy with the patient in the supine position directly located a CSF fistula in 19 patients only. In the other patients, the endoscopic examination revealed which nasal cavity had wetter and shiner nasal mucosa and permitted collection of transparent fluid in the nasopharynx but did not allow for precise visualization of the source of liquorrhea. Glucose concentration in the nasal discharge was higher than $30 \mathrm{mg} / \mathrm{dl}(1.7 \mathrm{mmol} / \mathrm{l})$ in all patients.

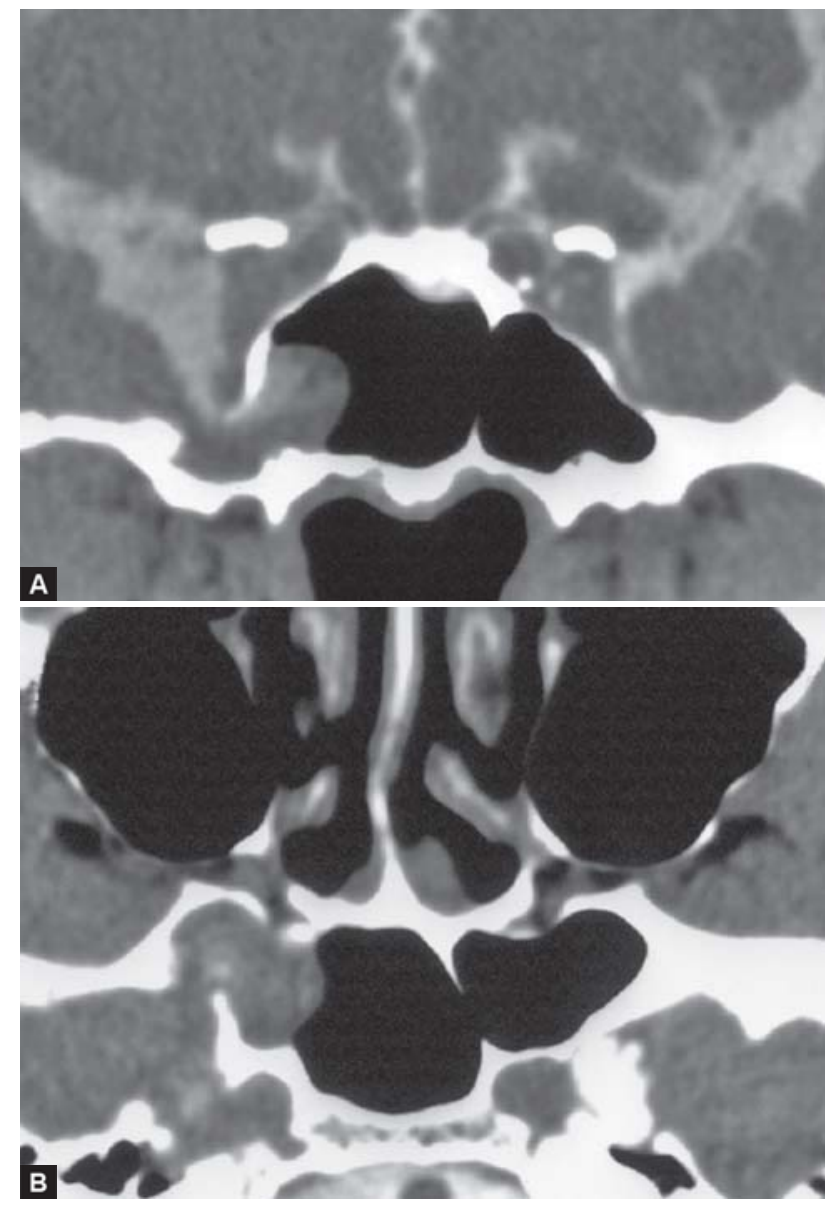

Figs 1 A and B: Axial (A) and coronal (B) computer tomographic scans from a patient with CSF rhinorrhea and a meningoencephalocele originating from a lateral extension of the right sphenoid sinus (so-called Sternberg's canal)
$\mathrm{CT}$ and $\mathrm{CT} / \mathrm{MR}$ R cisternography were able to detect the precise site of the fistula in 146 cases. In the 27 remaining cases, a bone defect itself was not visualized; however, local mucosal edema caused by irritation, collection of the CSF in specific sinuses, or ethmoidal cells suggested the fistula's location. A common finding in this series was extremely pneumatized sphenoid sinus which extended to the apex of the temporal pyramid. In 26 cases, a bony defect and/or meningo/encephalocele appearing to be a source of the CSF leak were found in the lateral wall of the overpneumatized sphenoid sinus (Figs $1 A$ and $B$ ).

\section{Surgical Technique}

Fistula repair was performed using endonasal endoscopic technique under general anesthesia. In all, 186 surgeries (173 primary attempts and 13 revisions) were carried out over the 12-year period. Lumbar puncture was usually performed in the operative room before the beginning of the surgery. Cerebrospinal pressure was measured and a lumbar drain was placed with care taken to avoid evacuation of significant amount of CSF. During the surgery, the drain was kept closed till the final stage of the fistula repair. This maintained release of the CSF into the nasal cavity and thus facilitated the fistula's location.

The surgical technique was always dependent on the individual fistula size and position. The operation began with careful endoscopic examination of the nasal cavity. Once the patient was in supine position, examination often reveal ed collection of fluid in the nasopharynx. In cases of rapid CSF leaks, preoperative decongestion of the nasal mucosa and tractions of the middle turbinate identified a CSF pathway either from the natural ostium of the sphenoid sinus, or from the natural ostium of the posterior ethmoid or in the area of the cribriform plate. Thus, in cases where CT data were not conclusive, intraoperative endoscopy allowed for approximate location of the bone defect and selection of tentative surgical technique.

In no case did, the operation begin with the middle turbinate resection. Fracture and maximal lateral rotation of the turbinate were enough for good visualization of a bone defect in the medial portion of the cribriform plate. For the fistulas located in the lateral part of the cribriform plate and fovea ethmoidal is, we rotated the middle turbinate medially and cut a wide opening of the ethmoid sinus using the straight $3.5 \mathrm{~mm}$ aggressive blade of a microdebrider. The steps for the sphenoid sinus fistula were lateralization of the middle turbinate, identification of the sphenoidal ostium, and its enlargement inferiorly and laterally by cutting downward with the 900 Kerisson punch. The CSF fistula could not be easily approached in the lateral wall of the overpneumatized sphenoid sinus, but even in that case, middle turbinate resection was not performed because this 
Spontaneous CSF Leaks and Meningoencephaloceles: Endoscopic Repair and Possible Etiology

would not provide any benefit. If needed, the turbinate was returned to its initial medial position for better visualization and easier access to the lateral part of the sinus.

It is imperative for successful fistula repair to remove mucosa from the edges of the bone defect. In case of meningocele, the latter was removed first, and then bone edges of the fistula were carefully skeletonized. Selection of a plastic material for the fistula repair was also dependent on size and position of the fistula. When the bony defect was precisely visualized, a piece of nasal septum cartilage or several layers of autogenous fat were placed intracranially inside and the defect which was then closed with a piece of autogenous fascia lata in on-lay fashion. Rotating middle turbinate flaps were used for closure of bone defects in the cribriform plate and fovea ethmoidal is. If direct visualization of the fistula with a $0^{\circ}$ endoscope was not possible, the walls of the diseased sinus were deepithelized as much as possible and the sinus was obliterated with the autogenous fat. Obliteration of the cavity of the ethmoidal cells and medial displacement of the middle turbinate were performed in a way that did not obstruct the frontal sinus ostium. Septal cartilage was used for closure of large bone defects in the roof of the ethmoid, posterior walls of the sphenoid and the frontal sinus. The plastic materials used are listed in the Table 1. In the vast majority of patients, the fistula repair was achieved with a combination of different plastic materials. A mount of 0.5 or $1.0 \mathrm{cc}$ of Tissucol fibrin glue (162 cases) or BioGlue (24) was used for fixation of the flaps and plastic materials. Two or three pieces of M erocel packs were placed in the nasal cavity at the end of the surgery.

Patients were allowed to stand on the day after surgery but they were asked to avoid blowing their noses and coughing. The lumbar drain was kept for 5 to 6 days and CSF was evacuated by the drain at the rate of $5 \mathrm{ml}$ per hour. Packs were removed from the common nasal meatus on the 3rd day and from the upper/middle meatus on the 5th or 6th day.

\section{RESULTS}

The locations of CSF fistulas in this series of patients are listed in the Table 2. In 70 cases, the bone defect was found in the cribriform plate, and the fovea ethmoidalis was the source of CSF rhinorrhea in 50 patients. A mong the latter, defect in the anterior part of the ethmoidal roof was found in 27 cases and, in the posterior part, in 23 cases. In five patients, source of the leak was also situated somewhere in the ethmoidal roof but precise intraoperative location of the

Table 1: Plastic materials used for CSF fistulas repair

\begin{tabular}{lr} 
Autogenous fascia lata alone & 38 \\
Rotating middle turbinate/nasal septum mucosal flap & 8 \\
Septal cartilage + fascia lata & 46 \\
Fascia lata + abdominal fat & 81 \\
\hline
\end{tabular}

Table 2: Location of CSF fistulas and results of surgical repair

\begin{tabular}{lcl}
\hline Location of CSF fistulas & $\begin{array}{l}\text { Total } \\
\text { number }\end{array}$ & $\begin{array}{l}\text { Recurrence, } \\
\text { revision } \\
\text { surgery }\end{array}$ \\
\hline Cribriform plate & 70 & 2 \\
Roof of anterior ethmoid & 27 & 1 \\
Roof of posterior ethmoid & 23 & None \\
Roof of ethmoid (without precise & 5 & None \\
intraoperative location) & 26 & 5 \\
Lateral recess of sphenoid sinus & 19 & None \\
Posterior or superior & 3 & \\
wall of sphenoid sinus & 3 & 8 \\
Posterior wall of frontal sinus & 173 & \\
\hline Total & & \\
\hline
\end{tabular}

fistula site was not possible because of the absence of active liquorrhea at the time of surgery. In the other 45 cases, CSF rhinorrhea originated from the sphenoid sinus; among these fistula was revealed on the posterior or superior wall of the sinus in 19 patients, in the overpneumatized lateral part of the sinus-in the remaining 26. An extremely pneumatized sphenoid sinus was a quite common finding. This type of pneumatisation was present in 26 patients and, in 21 of them, source of the leak was found in the lateral sinus wall. There were only three cases of CSF leak from the posterior wall of the frontal sinus. M eningo/encephaloceles protruding through the bone defect was detected in 61 cases; in 25, it was located in the lateral wall of the sphenoid sinus (al ways in a deep lateral recess), and in 35, it situated on the upper wall of the ethmoid. In the remaining case, encephalocele originated from the posterior frontal sinus wall.

In 94 patients, size of the skull base bone defect was not greater than $3 \mathrm{~mm}$, in 53, it was between 4 and $7 \mathrm{~mm}$; and in another 26 patients, it was larger, between 8 and $14 \mathrm{~mm}$. One patient had two small fistulas in a very thin posterior wall of large sphenoid sinus extending beneath the sella turcica (Fig. 2). The absence of an active CSF leak at the time of the surgery and/or the bone defect hidden site

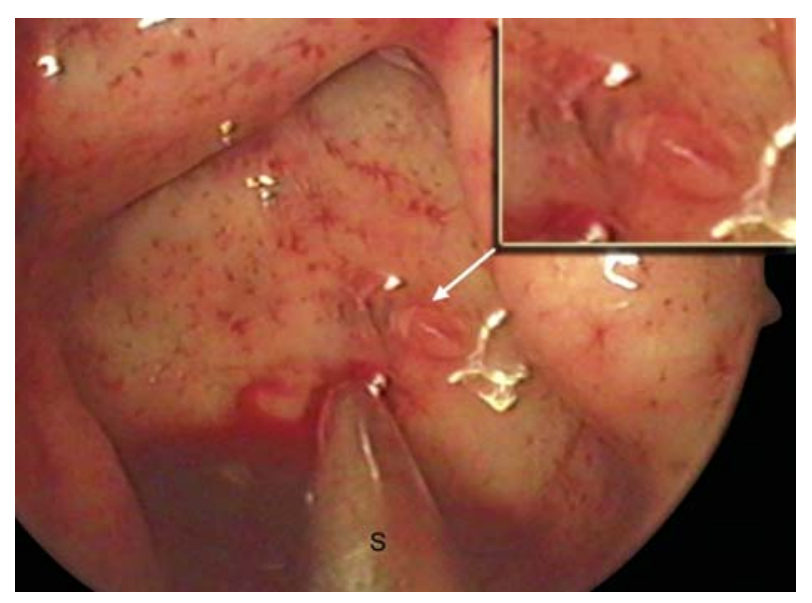

Fig. 2: Intraoperative view $\left(0^{\circ} \mathrm{scope}\right)$. O ne of the two leaking fistulas in the posterior sphenoid sinus wall is under the suction tip (S), and the second is indicated with the arrow 


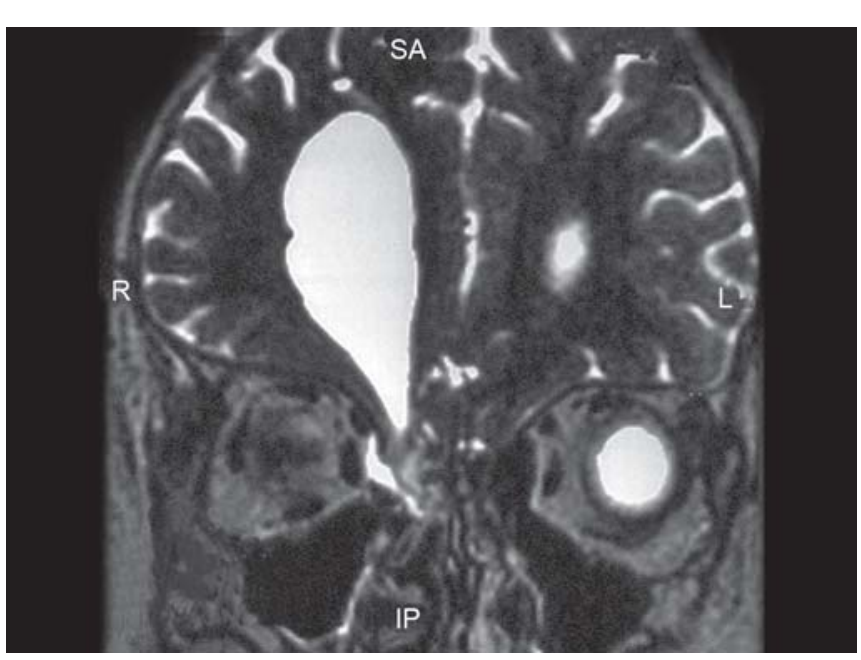

Fig. 3: MRI scan (coronal view) from a patient with a CSF fistula connected with significantly enlarged lateral ventriculum

prevented direct visualization and estimation of the fistula size in 32 patients.

Follow-up varied from 8 months to 11 years. Immediate results were good in all patients, but later during follow-up 13 patients devel oped recurrent CSF rhinorrhea. The source of recurrent leak was situated in a deep lateral recess of the sphenoid sinus in nine patients. Those were the cases where skull base defect was not adequately approached and managed during primary surgical intervention through the direct paraseptal approach. Endoscopic revision surgery was attempted in the same fashion in 12 cases, one patient refused further surgical treatment. Two revision surgeries failed again and the leak recurred after few months. One of these patients underwent third attempt of the fistula closure through the transcranial approach. This was a unique case where fistula had been connected with significantly dilated lateral ventriculum (Fig. 3).

\section{DISCUSSION}

Head trauma is the most common cause for CSF leak and majority of trauma-related CSF leaks resolve without surgical intervention. Persistent CSF rhinorrhea is potentially lethal. As it may lead to meningitis or brain abscess, surgical treatment is often required. Since St Clair Thompson's first description of 20 cases of nontraumatic rhinoliquorrhea in the late $1800 \mathrm{~s},{ }^{3}$ patients with primary (spontaneous) CSF leak have presented both a diagnostic and therapeutic challenge. Spontaneous CSF rhinorrhea has been always considered as an infrequent entity that constitutes not more than 3 to $4 \%$ of all CSF leaks. Review articles on spontaneous CSF leaks did not cited more than 10 cases. $^{4}$ In 1960, CE Troland found only 33 cases of surgically treated primary CSF leaks mentioned in the world literature. ${ }^{5}$ Twenty-five years later, J L Hubbard et al ${ }^{6}$ presented a personal series of nine cases; in $1991 \mathrm{NS}^{\mathrm{T}} \mathrm{Tolley}^{7}$ reported 10 more cases. With 161 cases, paper by B Schick et $\mathrm{al}^{8}$ remained probably the largest series of surgically treated CSF leaks; of these, 70 were caused by trauma, 36 by tumors and 47 were complications of paranasal sinus surgery. O nly 8 leaks in this series were spontaneous, caused by occult malformations of the skull base. These malformations are thought to be one of the main factors predisposing to a spontaneous CSF leak. ${ }^{9}$ The second risk factor for development of spontaneous rhinoliquorrhea is obesity. Overweight increases intraabdominal and intrathoracic pressure. It may affect blood circulation in cranial venous collectors and lead to development of permanent benign intracranial hypertension. ${ }^{10,11}$

The more recent reports have demonstrated both increasing preval ence of spontaneous CSF rhinoliquorrhea, and higher rate of spontaneous CSF leaks from fistulas of another etiology. In 2005, C Dunn et al ${ }^{12}$ reported that in a series of 29 consecutive patients with CSF, rhinorrhea was spontaneous in 10. O ur personal experience also indicates that spontaneous CSF rhinorrhea is not a condition as infrequent as was formerly believed. Of the 69 CSF leak patients admitted during 3-year period, 25 were diagnosed with spontaneous CSF rhinorrhea. ${ }^{13}$

It is essential to precisely locate the CSF fistula site whenever surgical closure is considered. This is why distribution of the sites of spontaneous CSF fistulas needs to be discussed. It was a common opinion that the cribriform plate and ethmoidal roof were the sites of the fistula location in most patients and the sphenoid sinus was rarely implicated in spontaneous CSF fistula. In contrast, our series as well as some other reports ${ }^{14,15}$ show that aside from the other innate skull base malformations, which occur in the area of the cribriform plate, posterior and lateral walls of the sphenoid sinus and particularly its lateral extensions are quite typical sitesfrom which spontaneous CSF leak may appear. Extreme pneumatization of the sphenoid sinus may cause bone resorption and prolapse of the dura through the persistent lateral craniopharyngeal (Sternberg's) canal - one of theleast resistant point at the skull base and the sphenoid bone. A meningo/encephalocele may originate from this bony dehiscence in the overpneumatized area and this pathogenic mechanism should beconsidered importantetiological factors for spontaneous CSF leak development. ${ }^{16-20}$

In our group, extensive type of the sphenoid sinus pneumatization was revealed in 26 patients, and a meningo/ encephalocele protruding through the bone defect in the lateral part of the overpneumatized sinus was found in 23 cases. It seems that in this particular type of CSF fistula, presence of obesity and intracranial hypertension is not obligatory for development of CSF rhinorrhea. Of the 173 study group patients, 71 had increased body mass index, and in 14 patients with overpneumatized sphenoid sinus cerebrospinal pressure was normal. 
Spontaneous CSF Leaks and Meningoencephaloceles: Endoscopic Repair and Possible Etiology

Some cases of spontaneous CSF rhinorrhea may be due to an empty sella turcica. In this case, an intrasellar arachnoidocele can compress the pituitary gland against the posterior sphenoid sinus wall, erode the floor and lead to CSF rhinorrhea through the eroded sinus wall. The last two etiological factors may coexist. The combination of extremely pneumatized sphenoid sinus and empty sella syndrome theoretically increases the risk of developing CSF rhinorrhea. ${ }^{21}$ This can be illustrated by the case in our series, where two small fistulas were found in the thin eroded posterior wall of the sphenoid sinus.

$M$ ost of the publications dealing with endonasal surgical treatment of CSF rhinorrhea reported efficacy of the method to be 85 to $100 \%, 1,2,8,19,22$ but most of these publications dealt with all types of CSF leaks. Reports on endonasal surgical treatment of spontaneous leaks are still rare. However, spontaneous CSF rhinorrhea often presents the most challenging cases. Fistulas located in the lateral part of a large sphenoid sinus are extremely difficult to locate and repair with either endoscopic endonasal or transcranial approach. In our series, 165 patients were treated successfully with the first attempt and five more recovered after revision endoscopic surgery. Success rate after initial surgery was $95.4 \%$ and overall success rate was $97.7 \%$.

\section{CONCLUSION}

Spontaneous CSF rhinorrhea is a clinical entity difficult to manage. Etiological factors of this disease may include obesity, innate skull base malformations, overpneumatized sphenoid sinus, particularly its lateral extensions and empty sella syndrome. The possible role of these factors or their combinations must be considered when planning adequate therapy for spontaneous CSF leak. A II the patients require surgical intervention and the endonasal endoscopic approach appears to be the best option. As there is no universal technique, specific surgery and choice of plastic materials depend on the fistula's size and location. H owever, treatment of CSF fistulas in the lateral part of the sphenoid sinus needs further perfectioning.

\section{REFERENCES}

1. Gjuric M, K eimer H, Goeede U, Wigand ME. Endonasal endoscopic closure of cerebrospinal fluid fistulas at the anterior cranial base. A nn Otol Rhinol Laryngol 1996;105:620-23.

2. Lanza DC, O'B rien DA, K ennedy DW. Endoscopic repair of cerebrospinal fluid fistulae and encephaloceles. L aryngoscope 1996;106:1119-25.
3. Thompson St C. The cerebrospinal fluid; its spontaneous escape from the nose. London: Cassel \& Co 1899.

4. Beckhard RN, Setzen M, Carras R. Primary spontaneous cerebrospinal fluid rhinorrhea. Otolaryngol Head Neck Surg 1991;104:425-32.

5. Troland CE. The surgical management of spontaneous cerebrospinal rhinorrhea. A rch Otolaryngol 1960;72:254-55.

6. Hubbard JL, M CD onald TJ, Pearson BW, et al. Spontaneous cerebrospinal fluid rhinorrhea. Evolving concepts in diagnosis and surgical management based on the $\mathrm{M}$ ayo $\mathrm{Clinic}$ experience from 1970 to 1981. N eurosurgery 1985;16:314-23.

7. Tolley NS. A clinical study of spontaneous CSF rhinorrhea. Rhinology 1991;29:223-30.

8. Schick B, W eber R, M osler P, K eerl R, Draf W. Long-term results of frontobasal duraplasty. HNO 1997;45:117-22.

9. Schick B, Draf W, Kahle G, Weber R, Wallenfang T. Occult malformations of the skull base. A rch Otolaryngol Head Neck Surg 1997;123:77-80.

10. Badia L, Loughran S, L und V. Primary spontaneous cerebrospinal fluid rhinorrhea and obesity. A m J Rhinol 2001;15:117-19.

11. Clark D, Bullock P, Hui T, Firth J. Benign intracranial hypertension: A cause of CSF rhinorrhea. J Neurol Neurosurg Psychiatry 1994;57:847-49.

12. Dunn CJ, Alaani A, J ohnson AP. Study on spontaneous fluid rhinorrhea: Its etiology and management. J Laryngol Otol 2005;119:12-15.

13. Lopatin AS, K apitanov DN, Potapov A A . Endonasal endoscopic repair of spontaneous cerebrospinal fluid leaks. A rch Otolaryngol Head N eck Surg 2003;129:859-63.

14. Shetty PG, Shroff M M, Fatterpekar GM, Sahani DV, K irtane $M V$. A retrospective analysis of spontaneous sphenoid sinus fistula: MR and CT findings. A m J Neuroradiol 2000;21: 337-42.

15. Shetty PG, Shroff M M , Sahani DV, K irtane M V. Evaluation of $\mathrm{CT}$ and MR cisternography in the diagnosis of cerebrospinal fluid fistula. A m J Neuroradiol 1998;19:633-39.

16. Sternberg $M$. Ein bisher noch nicht beschriebener $K$ anal im Keilbein des M enschen. A nat A nz 1888;3:225-53.

17. Radoievitch S, J ovanovitch S. C ruveilhier-Sternberg embryonal canal (lateral craniopharyngeal canal) and its vestiges in the adult human. Rev Laryngol Otol Rhinol 1956;77(3-4):223-32.

18. Schick B, B rors D, Prescher A. Sternberg's canal-cause of congenital sphenoidal meningocele. Eur A rch Otorhinolaryngol 2000;257:430-32.

19. Castel nuovo P, Dallan I, Pistochini A, B attaglia P, L ocatelli D, Bignami M. Endonasal endoscopic repair of Sternberg's canal cerebrospinal fluid leaks. Laryngoscope 2007;117:345-49.

20. Tomazic PV, Stammberger H. Spontaneous CSF-leaks and meningoencephaloceles in sphenoid sinus by persisting Sternberg's canal. R hinology 2009;47:369-74.

21. Schlosser RJ, Bolger WE. Spontaneous nasal cerebrospinal fluid leaks and empty sella syndrome: A clinical association. Am J Rhinol 2003;17:91-96.

22. Castelnuovo P, M auri S, Locatelli D, Emanuelli E, Delu G, Giulio GD. Endoscopic repair of cerebrospinal fluid rhinorrhea: L earning from our failures. A m J Rhinol 2001;15:333-42. 